\title{
Differences between DSM-IV and DSM-5 as applied to general adult psychiatry
}

\author{
R. Murphy ${ }^{1,2, *}$ and B. Hallahan ${ }^{1,2}$ \\ ${ }^{1}$ Department of Psychiatry, Clinical Science Institute, National University of Ireland Galway, Galway, Ireland \\ 2 Department of Psychiatry, University Hospital Galway, Roscommon Mental Health Services, Galway, Ireland \\ First published online 2 November 2015
}

\section{Introduction}

The Diagnostic and Statistical Manual of Mental Disorders-5 (DSM-5) was published on 18 May 2013 (American Psychiatric Association, 2013). The revision of the previous edition was a 14-year process that involved amendment of diagnostic criteria, the addition of new disorders, subtypes and specifiers and the deletion of certain previous DSM-IV disorders. The groups tasked with revising the DSM and indeed the International Classification of Diseases (ICD) [the operational system of the World Health Organisation (WHO)] systems (for the soon to be published ICD-11) shared an overarching goal of harmonizing the two classifications as much as possible (American Psychiatric Association, 1994). Four principles guided the draft revisions of DSM-5 (1): (1) the DSM-5 is primarily intended to be a manual for use by clinicians and revisions must be feasible for routine clinical practice; (2) recommendations for revisions should be guided by research evidence; (3) where possible, continuity should be maintained with previous editions of DSM; and (4) no prior constraints should be placed on the degree of change possible between the previous and newest edition of DSM.

\section{General points}

A number of key changes are now found in the DSM-5 (Roberts and Louie, 2014). In an attempt to enhance diagnostic specificity and increase clinician utility, the DSM-5 replaces the previous not otherwise specified (NOS) designation with two options for clinical use, 'other specified disorder' and 'unspecified disorder'. The 'other specified disorder' category allows the clinician to communicate the specific reason why the presentation does not meet criteria for a diagnosis within a diagnostic class, whereas 'unspecified disorder' can be used if the clinician does not wish to specify the reason. The DSM-5 introduces the category 'another medical condition' that now replaces 'general medical condition' where relevant across all disorders.

\footnotetext{
* Address for correspondence: Dr R. Murphy, Department of Psychiatry, Clinical Science Institute, National University of Ireland Galway, Galway, Ireland.

(Email: ruthannmurphy1@gmail.com)
}

Cultural factors have now been given increased relevance and importance compared with previous editions of DSM. Section III of DSM-5 introduces a chapter, 'Cultural formulation' that details a discussion of culture as it pertains to various diagnoses. The construct of the culture-bound syndrome has been replaced by three concepts that aim to offer greater clinical utility and suggest cultural ways of understanding and describing illness experiences cultural syndrome, cultural idiom of distress and cultural explanation or perceived cause. The DSM-5 has moved to a non-axial documentation of diagnosis (formerly Axes I, II and III) with separate notations for important psychosocial and contextual factors (formerly Axis IV) and disability (formerly Axis V) with the Global Assessment of Functioning scale (formerly in Axis V) no longer present. This approach is consistent with established WHO and ICD guidelines, where an individual's functional status is considered separately from an associated diagnosis or symptom status.

DSM-5's restructuring of diagnostic categories through combination or consolidation of disorders aims to better reflect shared features or symptoms of related disorders and to better convey these inter-relationships within and across diagnostic chapters. The number of mental health disorders in DSM-5 has reduced from 172 in DSM-IV to 157, but also includes 15 new disorders. Two mental health disorders have been eliminated and 22 previous mental health disorders have been combined or consolidated within other mental health disorders. DSM-5 mental health disorders are now structured chronologically, from those diagnoses initially attained in early childhood, through to adolescence and then into adulthood. In total, there are 464 differences between DSM-IV and DSM-5; however, the vast majority of these are very minor, with the more significant of these as they pertain to general adult psychiatry (predominantly) discussed below.

\section{Mental health disorders}

\section{Neurodevelopmental disorders}

There are several significant differences evident in DSM-5 compared with DSM-IV in this chapter. 
Mental disorders are consolidated around central domains of developmental delay in DSM-5. Examples of this include language disorder, which now includes expressive and mixed receptive-expressive language disorders, motor disorders which incorporates the developmental disorders of coordination disorder, stereotypic movement disorder and tic disorders (including Tourette's syndrome) and specific learning disorder, which now incorporates disorders related to the acquisition of academic skills such as reading disorder, mathematics disorder, disorder of written expression and learning disorder not otherwise.

This consolidation of disorders is also evident in relation to social cognitive disorders with autism spectrum disorder now including previous DSM-IV disorders of autistic disorder, Asperger's disorder, childhood disintegrative disorder and pervasive developmental disorder - NOS. The diagnostic criteria for attention-deficit hyperactivity disorder now allow for the application of this disorder across the lifespan of an individual and include a lower threshold for making the diagnosis. Intellectual developmental disorder replaces intellectual disability with assessment of adaptive functioning (practical, social and conceptual skills) rather than IQ scores now used to define severity of intellectual disorder. Other minor differences include the renaming of some disorders with phonological disorder now called speech sound disorder and stuttering now called childhood-onset fluency disorder.

\section{Schizophrenia spectrum and other psychotic disorders}

A number of significant differences between DSM-IV and DSM-5 are also present in this chapter. In relation to schizophrenia, a core symptom of a delusion, hallucination or disorganized speech has to be present for a diagnosis. In addition, there is no differentiation between second and third person auditory hallucinations and the presence of a bizarre delusion or commenting voice in themselves are insufficient for a diagnosis. In addition, the differentiation between the various subtypes of schizophrenia (e.g. hebephrenia, paranoid) no longer exists except for the catatonia subtype. Catatonia itself can now be diagnosed as a specifier for depressive, bipolar and psychotic disorders, as a separate diagnosis in the context of another medical condition or as another specified diagnosis. To meet diagnostic criteria for catatonia, a similar number of symptoms (3 out of 12) are required irrespective of primary medical or psychiatric condition unlike in DSM-IV, where different numbers of symptoms were required for diagnostic purposes.

In relation to schizoaffective disorder, DSM- 5 states that the mood disorder period must be present for the majority of the time ( $>50 \%)$, as compared with the time frame discussed in the previous edition of DSM as being 'for a substantial portion of the total duration of the illness'. This revision confirms schizoaffective disorder as a longitudinal instead of a cross-sectional diagnosis. For a diagnosis of a delusional disorder, delusions in DSM-5 can now include bizarre delusions as compared with DSM-IV; however, any delusion cannot markedly impair function or lead to obviously bizarre behaviour. In addition, shared psychotic disorder (i.e. shared delusional disorder) is no longer a separate diagnosis and is coded under the appropriate psychotic disorder (e.g. delusional disorder).

\section{Bipolar and related disorders}

Bipolar and related disorders are now situated in their own chapter, rather than combined with depressive disorders and appear following the chapter on schizophrenia and other psychotic disorder in recognition of the place of bipolar disorders as a bridge between the schizophrenia spectrum and the depressive disorders categories in terms of symptomatology, family history and genetics.

To enhance the accuracy in diagnosis and facilitate earlier detection in clinical settings, criterion A for manic and hypomanic episodes now includes an emphasis on changes in activity and energy as well as mood. In DSM-IV, the diagnosis of bipolar I disorder mixed episode required the individual to simultaneously meet full criteria for both manic and major depressive episodes. A new specifier, 'with mixed features' has been introduced that can apply to episodes of mania or hypomania when depressive features are present and also to episodes of depression when features of mania or hypomania are present. This specifier is applied if there are at least three manic or hypomanic symptoms occurring concurrently with at least five depressive symptoms. For manic, hypomanic and major depressive episodes, the DSM-5 has added the specifier, 'with anxious distress' to identify patients with anxiety symptoms that are not part of the bipolar disorder diagnostic criteria. This is defined as the presence of at least two of the following symptoms feeling keyed up or tense, feeling unusually restless, difficulty concentrating due to worry, fearing something awful may happen and fearing one may lose control of self.

Other specified bipolar and related disorders include categories for patients with a past history of major depressive disorder who meet criteria for hypomania except for duration ( $<4$ days), or for patients who have too few symptoms to meet the criteria for hypomania despite symptoms being present for a sufficient duration of time. 


\section{Depressive disorders}

Several new disorders have been included in this DSM-5 chapter. These include disruptive mood dysregulation, premenstrual dysphoric disorder and dysthymia. Disruptive mood dysregulation disorder has been introduced for children up to 12 years of age who exhibit persistent irritability and frequent episodes of extreme behavioural dyscontrol and has been introduced in an attempt to address concerns about the potential overdiagnosis and overtreatment of bipolar disorder in children. Its placement in the chapter on depressive disorders reflects, according to DSM-5, that children with these symptoms typically develop unipolar depressive disorders or anxiety disorders rather than bipolar disorder as they mature into adolescence or adulthood. Dysthymia includes both chronic major depressive disorder and the previous DSM-IV dysthymic disorder.

The criteria for major depressive disorder remain the same. However, a specifier for depressive disorders has been included, 'with anxious distress'. In addition, DSM-IV excluded a diagnosis of a major depressive episode for depressive symptoms lasting $<2$ months following the death of a loved one. This exclusion is omitted in DSM-5 and perhaps thus removes the implication that bereavement is up to 2 months in length when the duration is typically 1-2 years. Bereavement is recognized as a severe psychosocial stressor that can precipitate a major depressive episode in a vulnerable individual, with symptoms commonly beginning soon after the bereavement.

\section{Anxiety disorders}

Some DSM-IV disorders have been removed from and others added to the anxiety disorders diagnostic class in DSM-5. Obsessive-compulsive disorder (OCD), post-traumatic stress disorder (PTSD) and acute stress disorder have been moved into other diagnostic classes (see below). This reorganization emphasizes certain unique features such as compulsive behaviours in OCD and the exposure to a traumatic event in PTSD. Separation anxiety disorder and selective mutism have been moved into the DSM-5 anxiety disorder class (formerly under the chapter on, 'disorders usually first diagnosed in infancy, childhood or adolescence'). Other additional disorders in this chapter include substance/ medication-induced anxiety disorders and anxiety disorders due to another medical condition.

The diagnoses of agoraphobia, generalized anxiety disorder, separation anxiety disorder, specific phobia and social anxiety disorder now require symptoms to occur during a period of at least 6 months to meet diagnostic criteria in adults, which potentially minimizes the risk of over diagnosis of transient fears.
For agoraphobia, specific phobia and social anxiety disorder, these diagnoses no longer requires that individuals recognise that their anxiety is excessive or unreasonable, merely that the anxiety must be out of proportion to the actual danger or threat in the situation. Panic disorder and agoraphobia are now separate and independent disorders that may occur individually or together. Social anxiety disorder (formerly social phobia) describes marked fear or anxiety about one or more social situations in which the individual is exposed to possible scrutinizing by others, including social interactions, being observed and performing in front of others. Panic attacks are now described as either, 'expected' or 'unexpected' and are a specifier for all disorders.

\section{Obsessive-compulsive and related disorders}

This new diagnostic class not only includes OCD but also trichotillomania from the impulse-control disorders chapter, body dysmorphic disorder (BDD) from the somatoform disorders chapter, and new disorders including hoarding disorder, excoriation (skin picking) disorder and substance/medicationinduced obsessive-compulsive and related disorder. Specified obsessive-compulsive and related disorders include body-focused repetitive behavior disorder (nail biting, lip biting, cheek chewing) and obsessional jealousy.

The diagnosis of trichotillomania no longer requires noticeable hair loss or that hair pulling must result in pleasure. New specifiers have been included for several disorders, such as 'with excessive acquisition' for hoarding disorder, 'tic related' for OCD and 'with muscle dysphoria' for BDD. For BDD, the diagnostic criterion describing repetitive behaviours or mental acts in response to preoccupations with perceived defects or flaws in physical appearance has been added. The diagnoses of OCD, BDD and hoarding disorder now include an insight specifier that ranges from 'good or fair' to 'poor' to 'absent/delusional' allowing for greater distinction according to insight.

\section{Trauma and stressor-related disorders}

This is a new chapter in DSM-5 and reflects empirical evidence that demonstrates the variability in responses to traumatic or stressful events. This chapter also includes reactive attachment disorder and disinhibited social engagement disorder (formerly the indiscriminately social/disinhibited version of reactive attachment disorder).

The diagnostic criteria of PTSD have been altered significantly in DSM-5. The stressor criterion is more explicit, with the subjective reaction criterion eliminated and a PTSD specifier 'with dissociative 
symptoms' now included. The symptom clusters criteria have been expanded to four (re-experiencing, avoidance, persistent negative alterations in cognitions and mood and alterations in arousal and reactivity). The persistent negative alterations in cognitions and mood cluster now also include newer symptoms such as persistent negative emotional states with the arousal and reactivity cluster including irritable behaviour or angry outbursts and reckless or self-destructive behavior.

The qualifying traumatic events for acute stress disorder are similarly more explicit, indicating whether the events were experienced directly, witnessed or experience indirectly. The criterion that characterized the subjective reaction to the trauma has also been removed.

Adjustment disorders are conceptualized as heterogenous stress response syndromes that occur after exposure to a distressing (traumatic or nontraumatic) event and now include the following specifiers - with depressed mood, with anxiety, with mixed anxiety and depressed mood, with disturbance of conduct, with mixed disturbance of emotions and conduct and unspecified.

\section{Dissociative disorders}

Criteria for dissociative identity disorder (DID) have been further expanded and now include certain possession-form phenomena and functional neurological symptoms to account for more diverse presentations and specifically states that transitions in identity may be observable by others or self-reported and that individuals with DID may have recurrent gaps in recall for everyday events and not just for traumatic experiences. Dissociative amnesia now includes dissociative fugue as a specifier with fugue no longer a separate disorder. Depersonalization disorder has been revised to include derealization as both often co-occur.

\section{Somatic symptom and related disorders}

This chapter replaces the somatoform disorders of DSM-IV, reducing the number of disorders and subcategories and emphasizes the presence of distressing physical complaints coupled with abnormal thoughts and behaviours rather than focusing on the presence of medically unexplained somatic symptoms. The absence of an identified medical aetiology for the individual's physical complaints is no longer the focus of the somatic symptom and related disorders but rather how the individual interprets these physical complaints and functions with them. This classification defines disorders on the basis of positive symptoms - distressing somatic symptoms plus abnormal thoughts, feelings and behaviours in response to these symptoms.

Diagnoses of somatization disorder, hyponchondriasis, pain disorder and undifferentiated somatoform disorder have been removed. Somatic symptom disorder now differs from somatoform disorders in that it does not require that the symptoms be medically unexplained. Psychological factors affecting other medical conditions is a new disorder, formerly included in 'other conditions that may be a focus of clinical attention'. Individuals with high health anxiety without somatic symptoms (who would previously have received a diagnosis of hypochondriasis) now receive a diagnosis of illness anxiety disorder or somatic symptoms disorder (if somatic symptoms are present).

Conversion disorder (functional neurological symptom disorder) no longer requires that the symptom or deficit be unintentionally produced or feigned due to the difficulty associated with reliably assessing underlying motivations behind neurological symptoms. The criteria for diagnosis are modified to emphasize the essential importance of the neurological exam and in recognition that relevant psychological factors may not be demonstrable at the time of diagnosis. The presence of secondary gain is no longer used to make a diagnosis of a somatic symptom and related disorder. If evidence is present that symptoms are intentionally produced, the diagnosis of factitious disorder or malingering should be considered and applied, with factitious disorder now listed among somatic symptom and related disorders.

\section{Feeding and eating disorders}

This diagnostic class combines the previous diagnostic class of eating disorders with some of the childhood disorders, avoidant/restrictive food intake disorder (formerly feeding disorder of infancy or early childhood), pica and rumination disorder (both of which can now be diagnosed at any age). Binge eating disorder (previously included as eating disorder NOS) is now included as a named diagnosis. The only change in relation to this diagnosis is that the minimum average frequency of binge eating required has been reduced from at least twice weekly for 6 months to at least once weekly over the last 3 months.

Some minor changes in relation to the diagnosis of anorexia nervosa are present. Amenorrhoea is no longer required for diagnosis and patients can have either an overtly expressed fear of weight gain or exhibit persistent behavior that interferes with weight gain, rather than both symptoms being required. For a diagnosis of bulimia nervosa, the only change for diagnostic purposes is a reduction in the required 
minimum average frequency of binge eating and inappropriate compensatory behavior frequency from twice to once weekly.

\section{Elimination disorders}

No significant changes were made in relation to this diagnostic class in DSM-5.

\section{Sleep-wake disorders}

Numerous changes have been made to this diagnostic class. The distinction between primary and secondary insomnia is eliminated, with the diagnosis insomnia disorder now present. The diagnosis of primary hypersomnia has been replaced by hypersomnolence disorder. The diagnosis of narcolepsy now requires not only subjective symptoms such as sleep attacks/ cataplexy but also objective biological indicators such as hypocretin deficiency (as measured in the cerebrospinal fluid) and a rapid eye movement (REM) sleep latency of $\leqslant 15$ minutes (as measured on nocturnal sleep polysomnograhpy).

The four diagnoses of breathing-related sleep disorder are now separated into three disorders obstructive sleep apnoea, central sleep apnoea and sleep-related hypoventilation. Circadian rhythm sleep-wake disorder now includes advance sleep phase syndrome, irregular sleep-wake type and non-24 hour sleep-wake type with the jet lag specifier removed.

Disorders previously listed as individual parasomnias such as sleepwalking and sleep terrors have been grouped into non-REM sleep arousal disorder. Nightmare disorder has been modified to include specifiers, to account for associated medical psychiatric and sleep disorders that may co-occur. Finally, two new diagnoses have been added - REM sleep behaviour disorder and restless legs syndrome (thereby reducing the NOS diagnoses). The diagnostic criteria for substance/medication-induced sleep disorder is unchanged in DSM-5, however tobacco has been included as one of the substances that can cause this disorder.

\section{Sexual dysfunctions}

DSM-5 introduces several significant general changes in this diagnostic class, making the diagnosis of sexual dysfunctions more specific and distinguished from transient sexual difficulties. All DSM-5 sexual dysfunctions (except substance/medication induced) now require a minimum of 6 month duration, distinguishing transient from more persistent sexual dysfunction. In addition, severity specifiers have been included to rate distress as mild, moderate or severe.
Two new diagnoses have also been included, female sexual interest/arousal disorder (combining sexual desire and arousal disorders) and genito-pelvic pain/ penetration disorder (combining the diagnoses of vaginismus and dyspareunia). Sexual aversion disorder has been removed and if present is classified under other specified sexual dysfunction.

\section{Gender dysphoria}

This is a new diagnostic class and reflects a change in conceptualization of the disorder's defining features by emphasizing the phenomenon of 'gender incongruence' rather than cross-gender identification in DSM-IV's gender identity disorder. The emphasis in DSM-5 is on the symptomatic response to the gender non-conformity as opposed to the pathologizing of the condition itself. Thus, gender dysphoria is not considered either a sexual dysfunction or a paraphilia, unlike in DSM-IV where gender identity disorder was grouped with these conditions.

Two specific states of gender dysphoria are included, namely gender dysphoria in children and in adolescents/adults. The experienced gender incongruence and resulting gender dysphoria can take many forms. Gender dysphoria is thus considered to be a mulitcategory concept rather than a dichotomy and DSM-5 acknowledges the wide variation of genderincongruent conditions. The previous criterion cross-gender identification and aversion towards one's gender have been merged and the wording of the criteria, 'the other sex' has been replaced by 'some alternative gender'. The subtyping on the basis of sexual orientation has been removed and a posttransition specifier has been added, as many individuals after transition no longer meet the criteria for gender dysphoria.

\section{Substance-related and addictive disorders}

There has been an elimination of the distinction between substance abuse and dependence and are now grouped under substance use disorders, with inclusion of criteria for substance use disorder, accompanied by criteria for intoxication, withdrawal, substance/ medication-induced disorders and unspecified substance-induced disorders where relevant.

Significant modifications to this chapter include the addition of gambling disorder, tobacco abuse, cannabis and caffeine withdrawal. The criterion for recurrent legal problems has been removed and a new criterion of craving or a strong desire or urge to use has been added with the threshold for substance use disorder diagnosis in DSM-5 increased. The criteria for substance use disorders now requires the fulfilment of four factors impaired control, social impairment, risky use and 
pharmacological criteria (including tolerance and withdrawal symptoms). Severity of substance use disorders is based on the number of criteria attained $2-3=$ mild, $4-5=$ moderate, $\geqslant 6=$ severe. The DSM-IV specifier for a physiological subtype has been eliminated, as has the DSM-IV diagnosis of polysubstance dependence.

\section{Personality disorders}

No changes have been made to the core features found in this diagnostic class but DSM-5 section III does offer an alternative model for personality disorders. This section characterizes personality disorders by impairments in: personality functioning (A) - disturbances in self (identity, self-direction) and interpersonal functioning (empathy, intimacy), pathological personality traits (B), the presence of both $\mathrm{A}$ and $\mathrm{B}$ found throughout each specific personality disorder, pervasiveness of the personality disorder (C) and stability of the personality disorder (D). Exclusion criteria based on the presence of other mental disorders, the effect of psycho-active substances or behaviour related to normal development are described.

\section{Paraphilic disorders}

A clear distinction is now present between paraphilic disorder and paraphilia (not included in DSM-IV). For a diagnosis of a paraphilic disorder, one requires the presence of both a paraphilia (the description of specific sexual arousal and behaviour) and its consequences (causing distress or impairment to the individual or risk or harm to others) for diagnosis. A course specifier, 'in a controlled environment' and 'in remission' has been added to the diagnostic criteria for all paraphilic disorders and the word disorder to all the paraphilias.

\section{Discussion}

During both the development and publication of DSM-5 there have been numerous critics and criticisms related to various aspects of the evolution of the current edition of DSM. For example, Robert Spitzer (head of the DSM III task force) and Allen Frances (chair of DSM-IV task force) have been amongst the most vocal, criticizing the American Psychiatric Association (APA) and their preliminary mandating of the revision task force to a non-disclosure agreement (Woodruff, 2010). Following such disapproval this decision was subsequently revoked and a disclosure policy was put in place.

Further criticism included the documented involvement of $70 \%$ of the DSM- 5 task force with the pharmaceutical industry. The British Psychological Society stated that it had 'more concerns than plaudits' for the revised manual and expressed fundamental concerns regarding the development, personal impact and core assumptions of the diagnosis system believing that use of the newest edition would likely lead to medicalization of natural and normal responses to patient experiences (British Psychological Society, 2011). Additional concerns relate to new diagnoses such as disruptive mood dysregulation disorder and attenuated psychosis syndrome in DSM-5 lacking empirical support, that inter-rater reliability was low for many disorders and that several sections contained poorly written, confusing or contradictory information (Robbins et al. 2012).

Thomas Insel, director of the NIMH, the National Institute of Mental Health described DSM-5 as 'at best a dictionary and not a bible', criticizing the lack of reliability of DSM-5 compared with previous editions (Insel, 2013). He has advocated for an alternative approach to the direction of psychiatric research to allow disorders to be defined by their causes rather then their symptoms. Following this, the NIMH had expressed interest and openly discussed the possibility for a new classification system - Research Domain Criteria (RDoC). In May 2013 Insel issued a joint statement along with J.A. Lieberman (President of the APA) emphasizing that DSM-5 represents the best information currently available for clinical diagnosis of mental disorders and that patients, families and insurers can be confident that effective treatments are available and that ultimately the DSM is the key resource for delivering the best available care. DSM and RDoC represent complementary and not competing frameworks for characterizing diseases and disorders.

Following expressed concern from Brent Robbins (Associate Professor of Psychology in Pennsylvania) regarding an apprehension that grief could be deemed pathological, a footnote was added that subsequently differentiated grief and depression (Robbins, 2011). Allen Francis, Psychiatrist and Chair of the DSM-IV task force, summarized what he believed to be the DSM-5, 'ten worst changes'. These included the lowering of the diagnostic threshold for identifying generalized anxiety disorder with the possibility of over diagnosis and over medicalization of what previously was deemed to be normal everyday worries, the current categorization of first time once off substance abusers with long standing substance abusers, the introduction of new diagnosis disruptive mood dysregulation disorder with limited research, the inclusion of mild neurocognitive disorder and the new specific diagnosis of binge eating disorder with lowered threshold for diagnosis of same (Francis, 2012).

Despite these unenthusiastic comments, there have also been more optimistic views (Rief, 2013). Diagnostic systems by their nature are always time-limitied constructs where the need for revision is determined 
by the advancement in clinical research and the DSM is no exception. Therefore, diagnostic classification systems simply reflect the best possible international expert consensus regarding scientific evidence and the provision of interventions in healthcare systems at a given point in time. With regard to the revision process itself, this involved a 5-year collaboration including internationally accepted experts, consultants and advisors grouped by diagnostic domain, systematic involvment of all stakeholder groups including patients, advocacy groups, healthcare providers, interest groups and the public, dozens of working groups coupled with overarching task forces evaluating and discussing current scientific evidence before making the final proposals for a revision and eventually adopting them.

\section{Conclusion}

The DSM-5 was introduced after a lengthy revision period and is associated with multiple differences compared with the DSM-IV, with the introduction of several new diagnostic chapters, refinement of diagnoses for several disorders and in many cases consolidation of two or more previous disorders in DSM-IV into one disorder in DSM-5. Indeed very few disorders in DSM-IV are unchanged in DSM-5. Therefore, knowledge of this current edition of DSM is a necessity for clinicians and other mental health professionals. Despite the lengthy collaborative process involved in the development of DSM-5, multiple criticisms of DSM- 5 have been voiced. Critics have suggested that diagnoses have been attributed to normal responses to experiences, that there is a lack of empirical support regarding certain diagnoses and critics have also stated that certain members of the DSM-5 task force were inappropriately included in this task force. Notwithstanding these criticisms, the DSMs are the most widely used manuals for the diagnosis of mental disorders both for clinical and research purposes and consequently an awareness of the DSM-5 is important for both clinicians and researchers alike.

\section{Acknowledgement}

The authors would like to thank the staff of the Department of Psychiatry, National University of Ireland, Galway.

\section{Financial Support}

This research received no specific grant from any funding agency, commercial or not-for-profit sectors.

\section{Conflicts of Interest}

None.

\section{References}

American Psychiatric Association (1994). Diagnostic and Statistical Manual of Mental Disorders (DSM-IV), 4th edn. APA.

American Psychiatric Association (2013). Diagnostic and Statistical Manual of Mental Disorders (DSM-5), 5th edn. APA.

British Psychological Society (2011). Response to the American Psychiatric Association: DSM-5 development (http://apps.bps. org.uk/_publicationfiles/consultationresponses/DSM-5\% 202011\%20-\%20BPS\%20response.pdf). Accessed 26 August 2015.

Francis A (2012). DSM5 is a guide not a bible - ignore its ten worst changes. APA approval of DSM- 5 is a sad day for psychiatry (www.psychologytoday.com/blog/dsm5-indistress / 201212/dsm-5-is-guide-not-bible-ignore-its-tenworst-changes). Accessed 26 August 2015.

Insel T (2013). Transforming diagnosis. National Institute of Mental Health, May (www.nimh.nih.gov/about/director/ 2013/transforming-diagnosis.shtml). Accessed 26 August 2015.

Rief W (2013). DSM-5 pros and cons. Verhaltenstherapie 23, 280-285.

Robbins B (2011). Open letter to the DSM-5. www.ipetitions. $\mathrm{com} /$ petition/dsm5/.

Robbins B, Rockwell D, Raskin J, Farley F, Kamens S (2012). Coalition for DSM-5 reform (www.dsm5-reform.com). Accessed 26 August 2015.

Roberts LW, Louie AK (2014). (eds). Diagnosis and DSM-5. In Study guide to DSM-5, pp. 3-19. American Psychiatric Publishing: Arlington.

Woodruff J (2010). Rethinking mental illness (http:/ / www. pbs.org/newshour/bb/health-jan-june10-mentalillness_ 02-10/). Accessed 26 August 2015. 